\title{
Recipient S-CSCF Assured (RSA) Session Transfer for IMS based NGN
}

\author{
Changho Yun, A-Ra Cho, Seung-Geun Kim, Jong-Won Park and Yong-Kon Lim, Member, KIMICS
}

\begin{abstract}
A session transfer method, referred to as a Recipient Serving-call session control function (S-CSCF) Assured (RSA), is proposed in order to support both session mobility and consistency in IP multimedia subsystem (IMS) based next generation network (NGN). RSA session transfer simplifies a basic session transfer operation specified in [1] by using the user agent client (UAC) like characteristics of an S-CSCF. To show its efficiency, the session transfer delay and the traffic cost of RSA session transfer are investigated and compared with those of existing session transfer applications, including Assured and Consultative session transfers, by practically considering multiple session transfer failures. It is shown that RSA session transfer can further improve user experience by reducing session transfer delay as well as traffic cost than Assured and Consultative session transfers.
\end{abstract}

Index Terms - IMS, SIP, session transfer.

\section{INTRODUCTION}

A next generation network (NGN) is an IP based packet switched network that supports seamless multimedia services to many users from heterogeneous access networks via common service provisioning [2][3]. The IP multimedia subsystem (IMS), standardized by $3 \mathrm{GPP}$, is considered a common service platform for an NGN. IMS can provide multimedia services irrespective of users' location and flexibly introduces new services from third party application providers due to its standardized interfaces and service capabilities [4][5]. There are several IMS procedures to provide users with multimedia services. A specific IMS procedure is merely exchanging session initiation protocol (SIP) messages (i.e., SIP requests and responses) during a SIP dialogue.

A session transfer is a significant IMS procedure in terms of session mobility because it is useful in the case of connecting to third-party user equipment (UE) on the call or changing to more efficient equipment when entering another service areas (e.g. from a cellular phone to a notebook computer) [6]. Two session transfer applications, including Assured and
Consultative session transfers are specified in [1]. These two session transfers are advantageous in that they commonly guarantee session mobility as well as session consistency even in the occurrence of session transfer failure. Without loss of generality, it is always motivated to design a session transfer as simple as possible by reducing the number of SIP messages, compared with these two session transfers. This originates from that the exchange of multiple SIP messages before media flow can result in remarkable session setup delay and burden to server buffers in practice, thus deteriorating users' experience [7].

To the end, we propose a Recipient Serving-call session control function (S-CSCF) Assured (RSA) session transfer. RSA session transfer removes several SIP messages of a basic session transfer operation specified in [1] with the help of user agent client (UAC) like characteristics of an S-CSCF also specified in [8]: an S-CSCF can independently generate SIP requests on behalf of a UE. Furthermore, we consider general cases that a session transfer may fail such as selecting a busy UE or a UE with incompatible quality of service (QoS) parameters, and the occurrence of SIP response timeout. Accordingly, multiple session transfer trials are reflected in RSA session transfer. It is expected that RSA session transfer can reduce the delay of session transfer and corresponding traffic cost more than Assured and Consultative session transfers with maintaining session consistency in the case of multiple session transfer failures.

In this paper, we demonstrate an NGN architecture incorporated with IMS and explain a basic session establishment operation, which is a preliminary procedure of a session transfer, in Section 2. In Section 3 , we briefly outline existing session transfer applications, including Assured and Consultative session transfers. In Section 4, RSA session transfer is described. In Section 5, the performance of RSA session transfer is analyzed in terms of the session transfer delay $(S T D)$ and the session transfer traffic cost (STTC), and these are compared with those of Assured and Consultative session transfers. Finally, we conclude this paper in Section 6.

Manuscript received July 16, 2010; revised July 20, 2010; accepted July 30, 2010 .

Changho Yun is with the Department of Ocean Engineering Research, Korea Ocean Research \& Development Institute (KORDI), 305-343, Korea (Tel: +82-42-866-3834, Fax: +82-42-866-3819, Email: sgn0178@moeri.re.kr) 


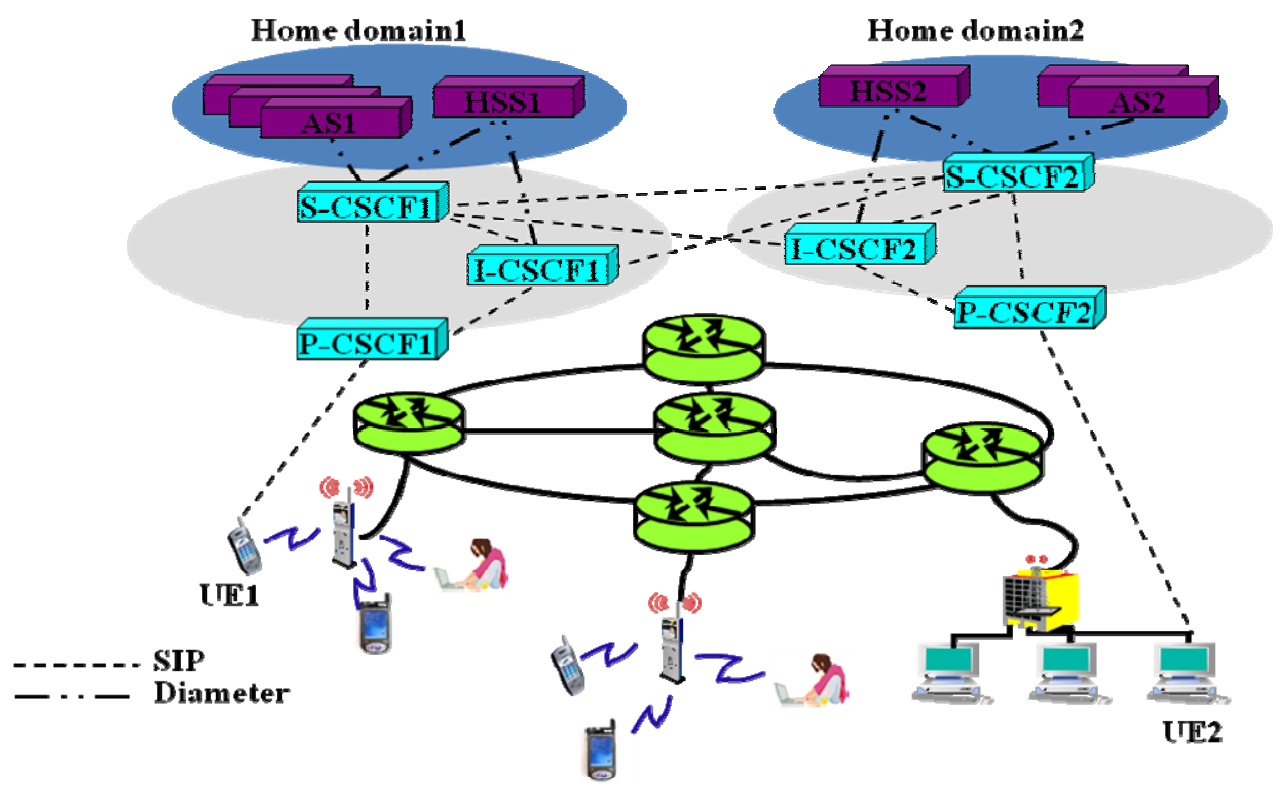

Fig. 1. An architecture of an NGN incorporated with IMS.

\section{AN IMS BASED NGN ARCHITECTURE AND IMS SESSION ESTABLISHMET}

An NGN incorporated with IMS consists of several functional entities (FEs) linked by standardized interfaces, as illustrated in Fig. 1 [9]. These FEs are various, including application servers (ASs), home subscriber servers (HSSs), S-CSCFs, proxy-CSCFs (P$\mathrm{CSCFs}$ ), interrogating-CSCFs (I-CSCFs), and S-CSCFs, UEs, and so forth. Their missions are described as follows [10][11].

- AS: A server providing UEs with several multimedia services (e.g., presence, push-to-talk, or conference).

- HSS: A server which stores UEs' registration databases which are the reference of an S-CSCF when it authenticates requested services of UEs.

- UE: An end point of IMS which can be located in various access networks, including general packet radio service (GPRS), Wi-Fi, digital subscriber loop (DSL), or even public switched telephone network (PSTN).

- P-CSCF: A SIP server which is the first contact point of a UE. It checks the IP security of a UE and forwards messages to S-CSCF.

- S-CSCF: A SIP server which authenticates a UE's registration and authorizes its request for a multimedia service.

- CSCF: A SIP server located in the edge of one home domain which is an IMS domain providing UEs with multimedia services via common service platform. It also helps to find the location of an S$\mathrm{CSCF}$ in another home domain.
The session establishment operation is simply exemplified as establishing a session between UE1 and UE2 for multimedia exchange, as illustrated in Fig. 1. Let's assume that two UEs belong to different home domain; home domain1 and home domain2, respectively. UE1 executes $\mathrm{P}-\mathrm{CSCF}$ query algorithm in order to find an adequate P-CSCF (P-CSCF1) and constructs IP-connectivity via P-CSCF1. Then, UE1 sends a REGISTER SIP message to P-CSCF1 which passes the SIP message to an I-CSCF of home domain 1 (I-CSCF1). I-CSCF1 finds a proper S-CSCF (SCSCF1) by referring to the HSS of home domain 1 (HSS1), and it forwards the Register SIP message to SCSCF1. Then, S-CSCF1 also queries HSS1 to authorize whether it provides UE1 with required service or not. UE2 also registers itself to its S-CSCF (S-CSCF2) in home domain 2 as what UE1 does.

If UE1 is registered by S-CSCF1, it sends an INVITE SIP message to UE2 in order to negotiate QoS parameters and reserve resource before multimedia exchange. The SIP message flows through P-CSCF1 and S-CSCF1, as well as an I-CSCF2, an S-CSCF2, and a P-CSCF2 in home domain2. If UE2 satisfies with the QoS parameters sent by UE1, it sends a 200 OK SIP message to UE1. If not, UE2 sends its desirable QoS parameters to UE1. This will continue until two UEs fully negotiate QoS parameters and finish resource reservation. More details of IMS session establishment operation can be described in [10][12]. 


\section{ASSURED AND CONSULTATIVE SESSION TRANSFERS}

Aforementioned, a session transfer is executed after a session is established between two UEs. These two UEs are primarily authorized to use several IMS services by registering to its S-CSCF. We narrow our scope to a session transfer such that all UEs are assumed to be registered to their S-CSCFs, respectively. Thus, we do not consider session registration of UEs upon describing session transfer. Let us consider two UEs currently establishing a session for media exchange in order to explain two session transfers. One UE, which initiates a session transfer to a third-party UE, is referred to as an Initiator. The remaining UE in an established session is referred to as a Recipient. The third-party UE, which is invited to establish a session with a Recipient, is referred to as a Target. It is common for the two session transfers to guarantee session mobility as well as consistency even in the occurrence of session transfer failure. In addition, we assume that an Initiator, a Recipient, and a Target have been already registered to its S-CSCF as well as contracted to use a REFER application.

\section{A. Assured Session Transfer}

SIP message to a Recipient, as shown in (1) of Fig. 2. Then, the Recipient sends an INVITE SIP message to a Target in order to establish a new session. Assured session transfer can be divided into two cases whether the INVITE SIP message is forwarded to the S-CSCF of an Initiator (S-CSCF2). Namely, the INVITE SIP message is passed to S-CSCF2 in Case I, and not in Case II, as illustrated in (1) of Fig. 2. In addition, if we consider the possibility of session transfer failure, Assured session transfer can be separated into three parts; a common part ((1) and (4) of Fig. 2), a session transfer success part ((2) of Fig. 2 ), and a session transfer failure part ((3) of Fig. 2). The session transfer is successful only if the Target accepts the invitation of the Recipient, which is illustrated as (2) in Fig. 2. The Recipient notifies the success of session transfer to the Initiator. Then, the Initiator disconnects a session with the Recipient. If the Recipient is rejected by the Target, it informs the Initiator of session transfer failure with the Target as shown in (3) of Fig. 2. Although the Target refuses to establish a session with the Recipient, the session between the Recipient and the Initiator still holds. The Initiator may try a session transfer to another Target UE\# such that a common part will be repeated, as shown in (4) of Fig. 2. All procedures of (4) of Fig. 2 is the same as those of (1) of Fig. 2. After executing (4) of Fig. 2, the remaining procedures can be followed as either (2) of Fig. 2 or (3) of Fig. 2 according to the response of the new target UE\#.

\section{B. Consultative Session Transfer}

Consultative session transfer consists of four parts; a session holding part, a common part ((1) and (4) of Fig. 3), a session transfer success part ((2) of Fig. 3), and a session transfer failure part ((3) of Fig. 3). In session holding part, an Initiator primarily holds a present session with a Recipient by sending a HOLD SIP message. In a common part, the Initiator establishes a new session with a Target for session transfer by sending an INVITE SIP message as shown in (1) of Fig. 3. In a session transfer success part, the Initiator succeeds to establish a new session with the Target as illustrated in (2) of Fig. 2. Then, the Initiator sends a REFER SIP message to the Target. As reverse as Assured session transfer, the Target sends an INVITE SIP message to the Recipient [1]. As a result, one more session setup is executed in Consultative session transfer, as shown in (2) of Fig. 3. After session transfer, as shown in (2) of Fig. 3. After the Target establishes a session with the Recipient by receiving an ACK SIP message from the Recipient, it sends a NOTIFY SIP message to the Initiator, as a response of the session transfer. Finally, the Initiator sends a BYE SIP message to both the Target and the Recipient in order to disconnect the session, respectively.

In a session transfer failure part, the Target sends a 4XX SIP message to the Initiator, as illustrated in (3) of Fig. 3. Although the session transfer fails, the session between the Initiator and Recipient is still alive because the session was held in the beginning of the session transfer. As Assured session transfer does, the Initiator can try a session transfer to another third-party UE\# as shown in (4) of Fig. 3 of which procedures are the same as those of (1) of Fig. 3. After executing (4) of Fig. 3, the remaining procedures are executed as either (2) in Fig. 3 or (3) in Fig. 3 with respect to the response of the new Target.

\section{RSA SESSION TRANSFER}

As we mentioned, RSA session transfer uses UAC like characteristics of an S-CSCF in a Recipient side. Accordingly, S-CSCF1 of a Recipient can send SIP requests to an Initiator or receives a response from the Initiator on behalf of its UE while executing session transfer. RSA session transfer also consists of three parts; a common part ((1) and (4) of Fig. 4), a session transfer success part ((2) of Fig. 4), and a session transfer failure part ((3) of Fig. 4). RSA session transfer can be described in detail as follows.

1. UE1 and UE2 currently establish a session and exchange multimedia, as illustrated in Fig. 4. Let's consider UE1, UE2, and UE3 as a Recipient, an Initiator, and a Target, respectively.

2. UE2 sends a REFER SIP message to UE1, which informs UE1 of sending an INVITE SIP message to UE3 as the same as Assured session transfer. 


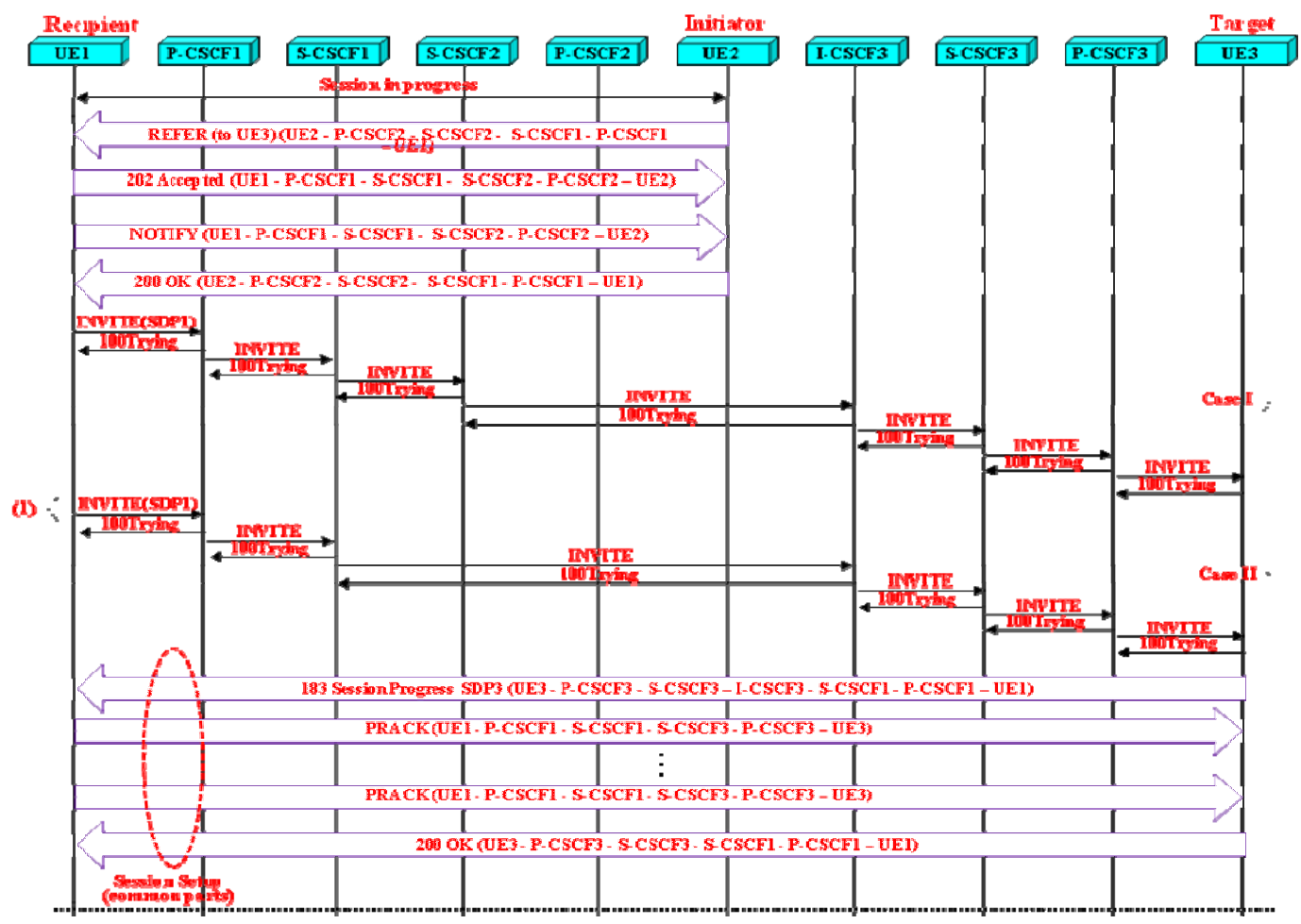

(2)

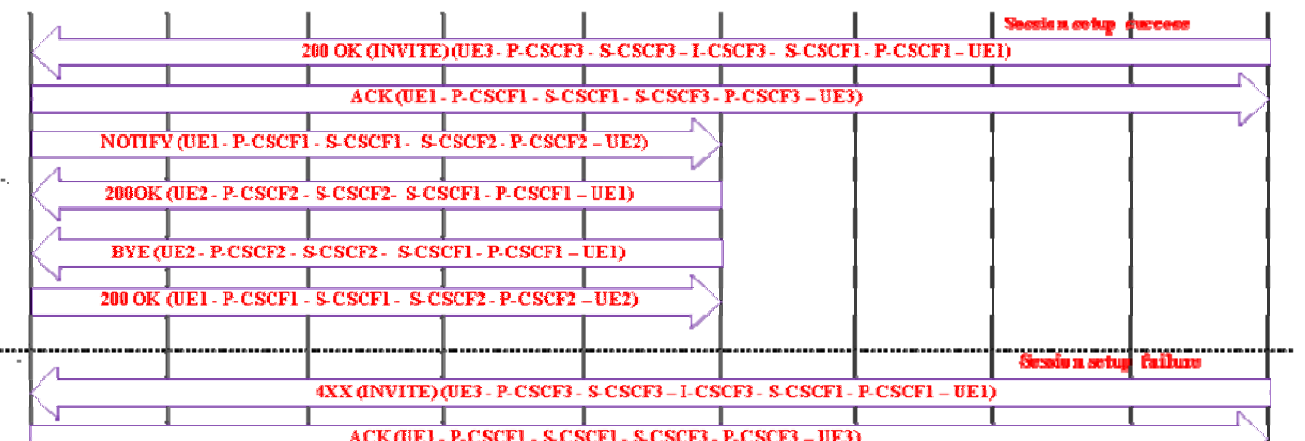

(3):

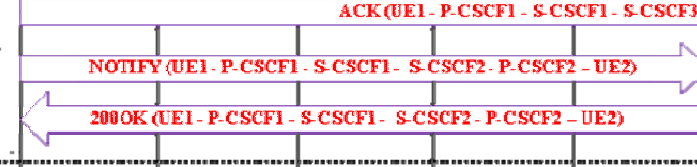

(4) :-
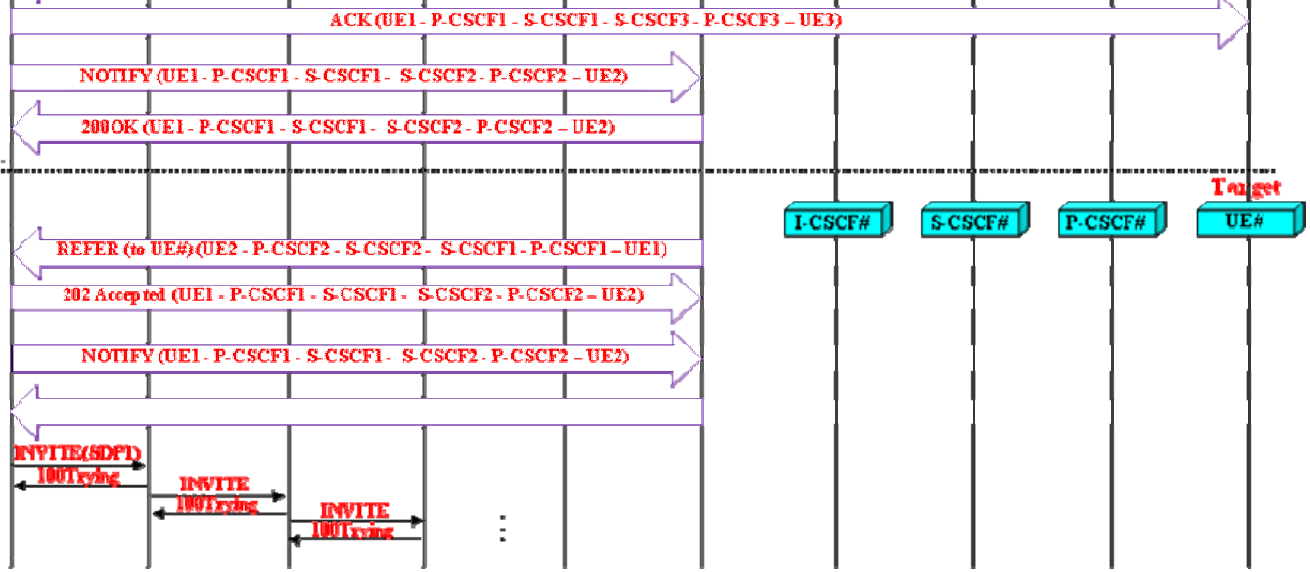

Fig. 2. Assured Session transfer. 


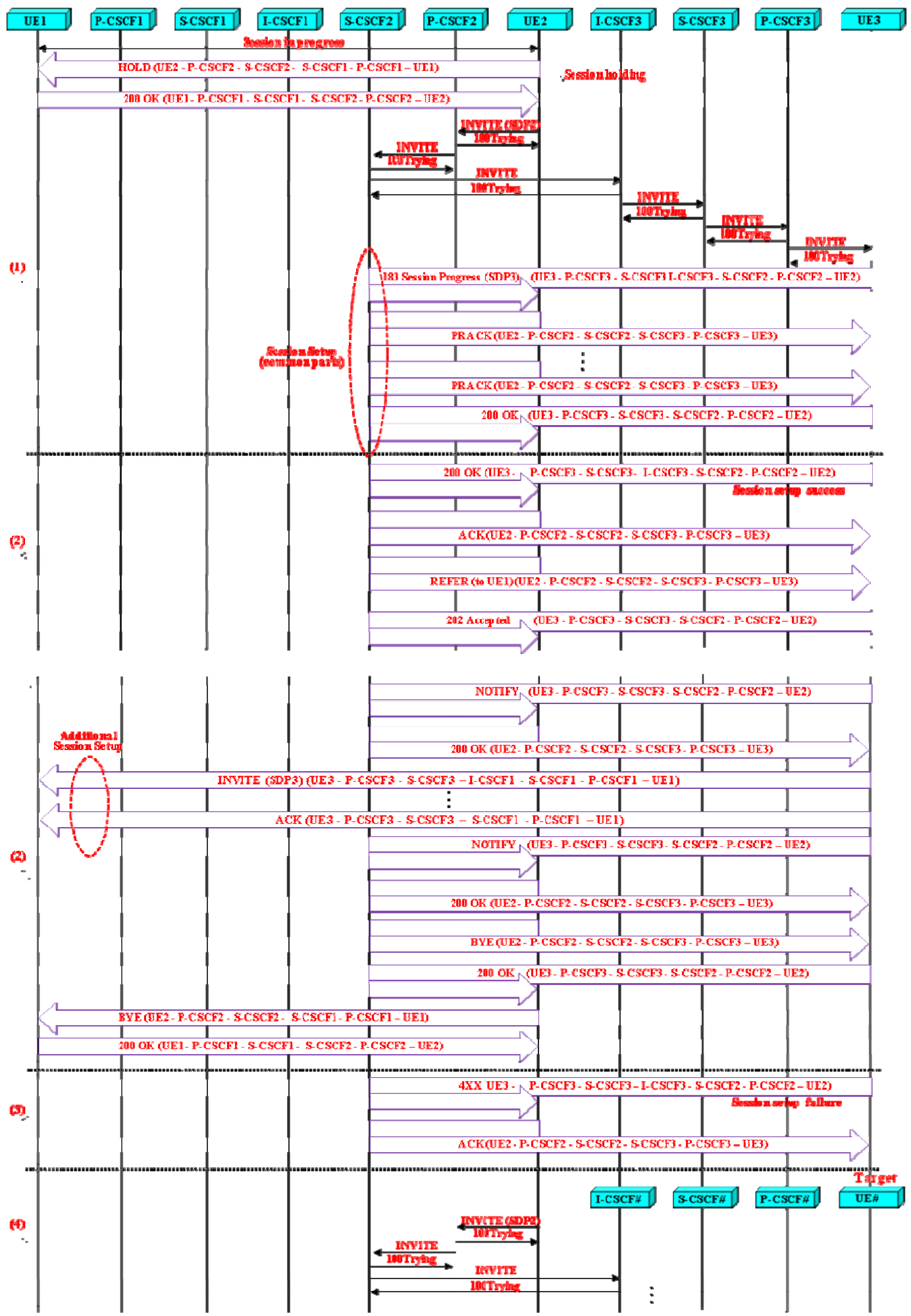

Fig. 3. Consultative Session transfer. 
3. S-CSCF1 just passes the REFER SIP message to UE1, waits for receiving an INVITE SIP message from UE1, and passes the SIP message to I-CSCF3, as specified as (1) in Fig. 4.

4. If UE3 accepts a session establishment with UE1, it sends a 200 OK SIP message as a response of UE1's invitation, as shown in (2) of Fig. 4. When S-CSCF1 receives the 200 OK SIP message, it sends a NOTIFY SIP message to UE2 instead of UE1. Then, S-CSCF1 continues to forward the 200 OK SIP message to UE1 as shown in the circle of (2) of Fig. 4. After UE1 receives the 200 OK SIP message, it sends an ACK SIP message to UE3 in order to finish a session setup. It is expected that this procedure can reduce the number of SIP messages as well as reduce the overall session transfer delay. UE2 sends a BYE SIP message to UE1 to terminate a session, and UE1 responses the SIP request by sending a 200 OK SIP message to UE2.

5. If S-CSCF1 receives a $4 X X$ SIP message from UE3 due to the failure of SDP parameter negotiation, it forwards the 4XX SIP messages to UE1 which will send an ACK SIP message to UE3 right after receiving the 4XX SIP message. Also, S-CSCF1sends a NOTIFY SIP message to UE2 in order to inform UE2 of session transfer failure as shown in (3) in Fig. 4.

6. In spite of session transfer failure, the session between UE1 and UE2 still holds, and this prevents session disconnection during a session transfer. UE1 knows the failure of session transfer by receiving the $4 \mathrm{XX}$ SIP message such that it does not send any response for the REFER SIP message.

7. If UE2 tries session transfer again to another UE\#, it repeatedly sends a REFER SIP message to UE1 by referring to UE\#, as shown in (4) of Fig. 4. All procedures illustrated in (4) of Fig. 4 is the same as those in (1) of Fig. 4.

8. If UE\# sends a 200 OK SIP message as a response of the INVITE SIP message, the remaining procedures are the same as those in step 4. If not, the procedures are equivalently implemented as steps 5 and 6 .

\section{PERFORMANCE ANALYSIS}

In this section, we investigate the STTC and STD of RSA session transfer, and compare them with those of Assured and Consultative session transfers through numerical analysis. To derive the $S T D_{X}$ and $S T T C_{X}$ of three session transfers where $X=\{R S A, A, C\}$ following assumptions are considered. $A$ and $C$ individually stand for Assured and Consultative session transfers.

- Session transfer can be successful after $N$ trials $(N \geq 1)$.

- More load can be added in S-CSCF1 of RSA session transfer than in that of Assured or Consultative session transfer due to extra works like determining whether S-CSCF1 sends a SIP like determining whether SCSCF1 sends a SIP like determining whether S-
CSCF1 sends a SIP message or not. In general, the processing capacity of servers such as S-CSCF is given much higher than that of UEs. Thus, the load of S-CSCF1 induced by the determination may not remarkably affect on RSA session transfer. Accordingly, the time for service control of all SCSCFs for RSA, Assured, and Consultative session transfers is assumed to be equivalent.

- As specified in [10][12], 12 SIP messages are considered to establish a session, including INVITE, 100 Trying, 183 Session Progress, PRACK, UPDATE, 180 Ringing, 200 OK, ACK SIP messages, and so forth.

- At simplicity, the time and the traffic cost to send each SIP message are commonly determined as unit time $T_{i}$ and unit traffic cost $C_{i}$ in order to obtain $S T D_{X}$ and $S T T C_{X}$, respectively as in [6].

\section{A. STTC of three session transfers}

Based the above assumptions, $S T T C_{R S A}$ for $N=1$ (i.e., the success of session transfer to UE3 at the first trial) is obtained by adding following traffic cost $(T C)$.

- $T C_{R S A}(1)$ is the traffic cost to execute all SIP procedures in (1) of Fig. 4. $T C_{R S A}(1)$ can be obtained as $74 C_{i}$ resulted from $5 C_{i}$ (REFER) $+5 C_{i}(202$ Accepted) $+5 C_{i}$ (NOTIFY) $+5 C_{i}$ (200 OK for NOTIFY) $+6 C_{i}$ (INVITE) $+6 C_{i}$ (100 Trying) $+6 C_{i}$ (183 Session Progress) $+5 C_{i}$ (PRACK) $+5 C_{i}(200$ OK for RPACK $)+5 C_{i}$ (UPDATE) $+5 C_{i}(200$ OK for UPDATE $)+6 C_{i}(180$ Ringing $)+5 C_{i}$ (PRACK) $+5 C_{i}$ (200 OK for RPACK).

- $T C_{R S A}(2)$ is the traffic cost to execute all SIP procedures in (2) of Fig. 4. $T C_{R S A}(2)$ can be obtained as $27 C_{i}$ resulted from $4 C_{i}$ (200 OK for INVITE from $\mathrm{UE} 2$ to S-CSCF1) $+3 C_{i}$ (NOTIFY) $+2 C_{i}(200$ OK for INVITE from S-CSCF1 to UE1) $+3 C_{i}(200$ OK for NOTIFY $)+5 C_{i}(\mathrm{ACK})+5 C_{i}(\mathrm{BYE})+5 C_{i}(200 \mathrm{OK}$ for BYE).

- $T C_{R S A}(3)$ is the traffic cost to execute all SIP procedures in (3) of Fig. 4. $T C_{R S A}(3)$ can be obtained as $17 C_{i}$ resulted from $4 C_{i}$ (4XX for INVITE from UE2 to $\mathrm{S}-\mathrm{CSCF} 1)+3 C_{i}$ (NOTIFY) $+2 C_{i}$ (4XX for INVITE from S-CSCF1 to UE1) $+3 C_{i}$ (200 OK for NOTIFY $)+5 C_{i}(\mathrm{ACK})$.

In case of $N=2$, the session transfer to UE3 at the first trial fails but that to UE\# at the second trial succeeds. Thus, $S T T C_{R S A}$ for $N=2$ is obtained as $T C_{R S A}(1)+$ $T C_{R S A}(3)+T C_{R S A}(4)+T C_{R S A}(2)$. Aforementioned, the SIP procedures of (1) of Fig. 4 is the same as those of (4) of Fig. 4 such that $S T T C_{R S A}$ for $N=2$ can be expressed as $2 T C_{R S A}(1)+T C_{R S A}(3)+T C_{R S A}(2)$. In case of $N=3$, session transfer can succeed at the third trial such that $S T T C_{R S A}$ can be represented as $3 T C_{R S A} \mathrm{TCRSA}(1)+2 T C_{R S A}(3)+$ $T C_{R S A}(2)\left(T C_{R S A}(1)+T C_{R S A}(3)+T C_{R S A}(4)+T C_{R S A}(3)+\right.$ $\left.T C_{R S A}(4)+T C_{R S A}(2)\right)$. As $N$ increases, $S T T C_{R S A}$ can be formulated as a function of $N$ as 


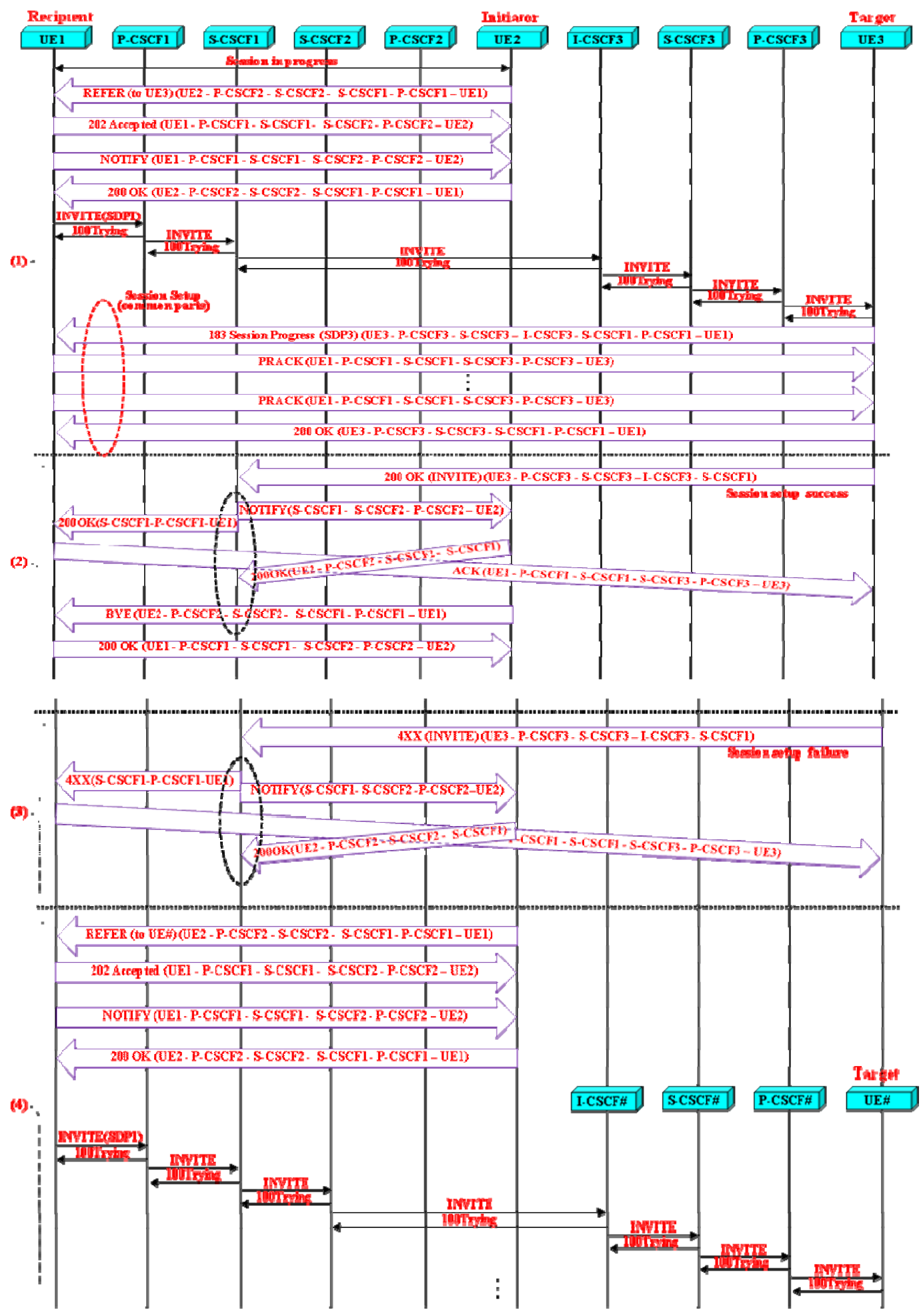

Fig. 4. RSA Session Transfer. 


$$
S T T C_{R S A}=N \cdot T C_{R S A}(1)+(N-1) \cdot T C_{R S A}(3)+T C_{R S A}(2) .
$$

By inserting the values of $T C_{R S A}(1), T C_{R S A}(2)$, and $T C_{R S A}(13)$ into Eq. (1), $S T T C_{R S A}$ can be expressed as

$$
\operatorname{STTC}_{R S A}=(91 N+10) \cdot C_{i} .
$$

In case of Assured session transfer, $T C_{A}(1), T C_{A}(2)$, and $T C_{A}(3)$, the counterparts of $T C_{R S A}(1), T C_{R S A}(2)$, and $T C_{R S A}(3)$, are individually obtained as follows.

- As shown in (1) of Fig. 2, $T C_{A}(1)$ for CaselI is the same as $T C_{R S A}(1)\left(74 C_{i}\right) . T C_{A}(1)$ for CaseI is $76 C_{i}$ because two more SIP messages (INVITE and 100 Trying SIP messages between S-CSCF1 and SCSCF2) is added.

- As shown in (2) of Fig. 2, $T C_{A}(2)$ can be obtained as $31 C_{i}$ resulted from $6 C_{i}(200 \mathrm{OK}$ for INVITE $)+5 C_{i}$ $(\mathrm{ACK})+5 C_{i}$ (NOTIFY) $+5 C_{i}$ (200 OK for NOTIFY) $+5 C_{i}$ (BYE) $+5 C_{i}(200 \mathrm{OK}$ for BYE).

- As shown in (3) of Fig. 2, $T C_{A}(3)$ can be obtained as $21 C_{i}$ resulted from $6 C_{i}$ (4XX for INVITE) $+5 C_{i}$ $(\mathrm{ACK})+5 C_{i}$ (NOTIFY) $+5 C_{i}(200 \mathrm{OK}$ for NOTIFY $)$.

As the same as $S T T C_{R S A}, S T T C_{A}$ is also obtained as a function of $N$ as $N \cdot T C_{A}(1)+(N-1) \cdot T C_{A}(3)+T C_{A}(2)$. Thus, $S T T C_{A}$ can be represented as

$$
\operatorname{STTC}_{A}=\left\{\begin{array}{l}
(97 N+10) \cdot C_{i}, \text { CaseI } \\
(95 N+10) \cdot C_{i}, \text { CaseII }
\end{array}\right.
$$

In case of Consultative session transfer, the $T C$ for session holding between UE2 and UE1 is $10 C_{i}\left(5 C_{i}\right.$ $(\mathrm{HOLD})+5 C_{i}\left(200 \mathrm{OK}\right.$ for HOLD)). $T C_{C}(1), T C_{C}(2)$, and $T C_{C}(3)$, the counterparts of $T C_{R S A}(1), T C_{R S A}(2)$, and $T C_{R S A}(3)$, are also respectively obtained as follows.

- As shown in (1) of Fig. 3, $T C_{C}(1)$ is $54 C_{i}$ originated from $6 C_{i}$ (INVITE) $+6 C_{i}$ (100 Trying) $+6 C_{i}(183$ Session Progress $)+5 C_{i}$ (PRACK) $+5 C_{i}(200 \mathrm{OK}$ for $\mathrm{RPACK})+5 C_{i}$ (UPDATE) $+5 C_{i}$ (200 OK for UPDATE $)+6 C_{i}(180$ Ringing $)+5 C_{i}$ (PRACK $)+5 C_{i}$ (200 OK for RPACK).

- As illustrated in (2) of Fig. 3, $T C_{C}(2)$ can be obtained as $126 C_{i}$ resulted from $6 C_{i}$ (200 OK for INVITE) + $5 C_{i}$ (ACK) $+5 C_{i}$ (REFER) $+5 C_{i}$ (202 Accepted) + $5 C_{i}$ (NOTIFY) $+5 C_{i}$ (200 OK for NOTIFY $)+6 C_{i}$ (INVITE) $+6 C_{i}$ (100 Trying) $+6 C_{i}(183$ Session Progress) $+5 C_{i}$ (PRACK) $+5 C_{i}(200 \mathrm{OK}$ for RPACK $)+5 C_{i}$ (UPDATE) $+5 C_{i}$ (200 OK for UPDATE $)+6 C_{i}$ (180 Ringing $)+5 C_{i}$ (PRACK) $+5 C_{i}$ $(200 \mathrm{OK}$ for RPACK $)+6 C_{i}(200 \mathrm{OK}$ for INVITE $)+$ $5 C_{i}$ (ACK) $+5 C_{i}$ (NOTIFY) $+5 C_{i}$ (200 OK for NOTIFY $)+5 C_{i}$ (BYE) $+5 C_{i}(200$ OK for BYE $)+$ $5 C_{i}$ (BYE) $+5 C_{i}$ (200 OK for BYE).

- As shown in (3) of Fig. 3, $T C_{C}(3)$ can be obtained as $11 C_{i}$ resulted from $6 C_{i}$ (4XX for INVITE) $+5 C_{i}$ (ACK).
$S T T C_{C}$ is also a function of $N$ and obtained as $10 C_{i}$ (TC for session holding) $+N \cdot T C_{C}(1)+(N-1) \cdot T C_{C}(3)+$ $T C_{C}(2)$. Thus, $S T T C_{C}$ is expressed as

$$
S T T C_{C}=(65 N+125) \cdot C_{i} \cdot
$$

Let us denote $V$ as session transfer traffic cost ratio which is obtained as $V=\frac{S T T C_{Y}}{S T T C_{R S A}}$. where $Y=\{A, C\}$.

\section{B. STD of three session transfers}

The coefficients of $S T D_{A}$ and $S T D_{C}$ are the same as those of $S T T C_{A}$ and $S T T C_{C}$ respectively because there are no SIP messages executed at the same time, as illustrated in Figs. 2 and 3. Accordingly, $S T D_{A}$ and $S T D_{C}$ are individually expressed as

$$
S T D_{A}=\left\{\begin{array}{l}
(97 N+10) \cdot T_{i}, \text { CaseI } \\
(95 N+10) \cdot T_{i}, \text { CaseII }
\end{array}\right.
$$

and

$$
S T D_{C}=(65 N+125) \cdot T_{i} \text {. }
$$

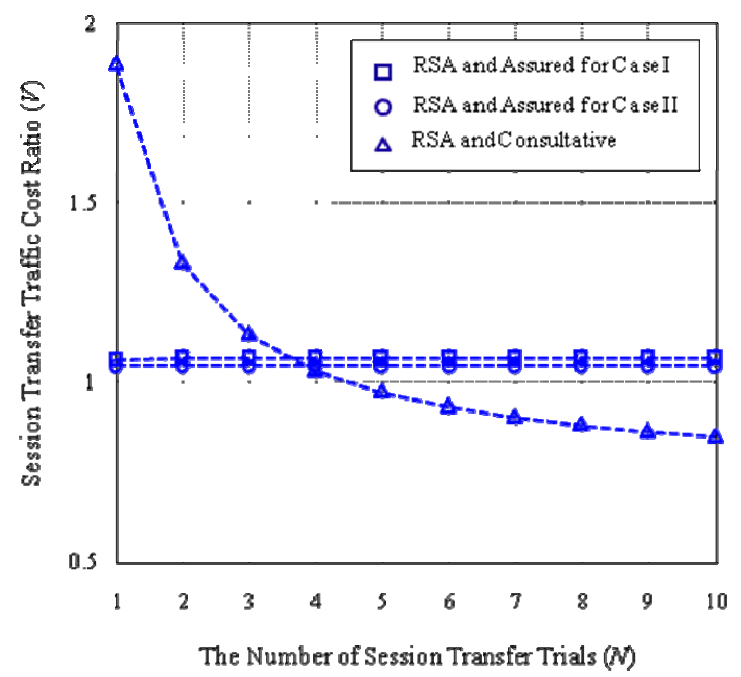

Fig. 5 Session transfer traffic cost ratio vs. the number of session transfer trials considering RSA, Assured, and Consultative session transfers.

In case of RSA session transfer, there are several SIP messages simultaneously executed such that following SIP messages need not to be considered upon deriving $S T D_{R S A}$.

- As shown in the circled part of (2) of Fig. 4, the time to send a 200 OK (from S-CSCF1 to UE1) and a ACK (from UE1 to UE3) SIP messages needs not to be counted because those SIP messages can be 
simultaneously dealt with a NOTIFY, a 200 OK (for NOTIFY), and a BYE SIP messages. Accordingly, $7 C_{i}$ can be reduced.

- As shown in the circled part of (3) of Fig. 4, the time to send a NOTIFY and a 200 OK (for NOTIFY) SIP messages are also not considered since the two SIP messages can be simultaneously dealt with a 4XX (from S-CSCF1 to UE1) and an ACK (from UE1 to UE3) SIP messages. Thus, $6 C_{i}$ can be reduced.

Let us denote the time to execute a common part, a session transfer success part, and a session transfer failure part of RSA session transfer as $D_{R S A}(1), D_{R S A}(2)$, and $D_{R S A}(3)$, respectively. As the same as $S T T C_{R S A}, S T D_{R S A}$ can be also formulated as a function of $N$, and thus expressed as $S T D_{R S A}=N \cdot D_{R S A}(1)+D_{R S A}(2)+(N-1)$. $D_{R S A}(3)$. The coefficient of $D_{R S A}(1)$ is nothing but that of $T C_{R S A}(1)$ as 74 . The coefficient of $D_{R S A}(2)$ is given as 20 resulted from 27 (the coefficient of $T C_{R S A}(2)$ )-7. Also, the coefficient of $D_{R S A}(3)$ is obtained as 11 resulted from 17 (the coefficient of $\left.T C_{R S A}(3)\right)-6$. Hence, $S T D_{R S A}$ can be expressed as

$$
S T D_{R S A}=(85 N+9) \cdot T_{i}
$$

Let us denote $S$ as session transfer delay ratio which is represented as $S=\frac{S T D_{Y}}{S T D_{R S A}}$. where $Y=\{A, C\}$.

\section{Results}

Overall, RSA session transfer can guarantee better session transfer delay performance than Assured session transfer, as shown in Fig. 5. RSA session transfer can reduce STTC (between Assured session transfer for CaseII and RSA session transfer) by at least $4 \%$ and STTC (between Assured session transfer for CaseI and RSA session transfer) by maximum 6\% regardless of $N$. The performance pattern between RSA and Assured session transfers still holds in the case of STD, as shown in Fig. 6. RSA session transfer can reduce STD (between Assured session transfer for CaseII and RSA session transfer) by at least $10 \%$ and STD (between Assured session transfer for CaseI and RSA session transfer) by maximum $12 \%$ regardless of $N$. It is noteworthy that the decrement of STD and STTC in RSA session transfer results from that RSA session transfer can basically reduce the number of overall SIP messages by that SCSCF1 sends a SIP request on behalf of UE1. In particular, STD performance improvement is further enhanced by that S-CSCF1 sends a SIP message right after sending another SIP message.

On the other hand, RSA session transfer can provide smaller STTC than Consultative session transfer when $N$ is small. For $N=1$, RSA session transfer can reduce STTC by almost $46 \%$. This performance superiority keeps when $N$ is around 4. As $N$ increases, Consultative session transfer outperforms RSA session transfer by avoiding to send several SIP messages in the occurrence of session transfer failure under HOLD-200 OK transaction. In the case of $S T D$, the performance pattern still keeps, but RSA session transfer can reduce $S T T C$ by almost $50 \%$ for $N=1$.

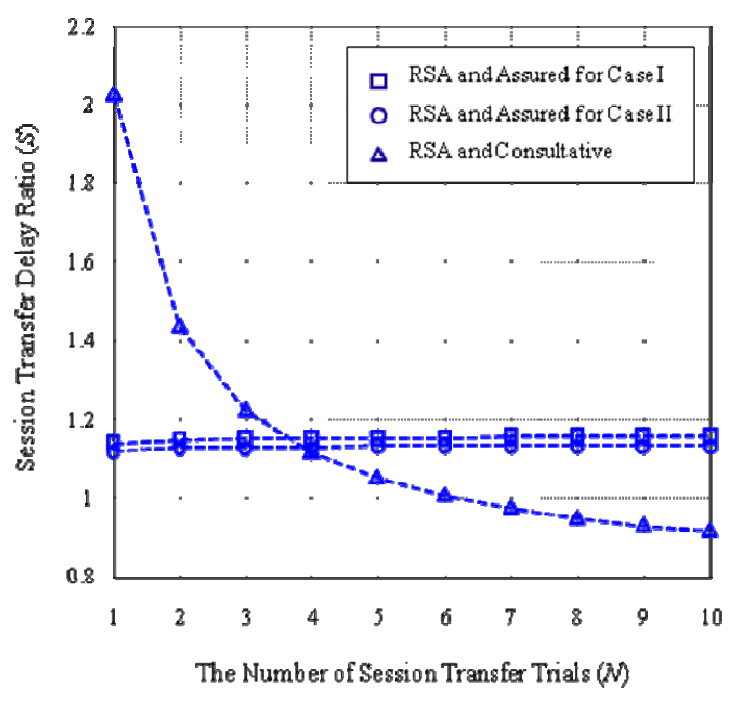

Fig. 6 Session transfer delay ratio vs. the number of session transfer trials considering RSA, Assured, and Consultative session transfers.

\section{CONCLUSIONS}

RSA session transfer has been proposed to simplify the exchange of multiple SIP messages of a session transfer operation specified in [1]. Accordingly, RSA session transfer can reduce session transfer delay, as well as corresponding traffic cost. In particular, session transfer delay and session transfer cost of RSA session transfer is analyzed and compared with Assured and Consultative session transfers. It is shown that RSA session transfer can support lower session transfer delay and traffic cost than Assured session transfer considering the multiple session transfer failures. Also, it outperforms Consultative session transfers under smaller session transfer failures. It is expected that RSA session transfer can be practically employed to various IMS services which are session delay-sensitive but pursue session mobility and SIP signaling efficiency. This is still ongoing work to enhance users' experience in IMS. Further works need to be considered in the sequel of this paper, including simulations for actual implementation by considering memory query and response time of servers under multiusers' access case. 


\section{ACKNOWLEDGMENT}

This work was conducted as a part of the research projects of "Development of marine RF based ad-hoc network for ship" financially supported by the Ministry of Land, Transport and Maritime Affairs (MLTM) of Korea.

\section{REFERENCES}

[1] 3GPP Definition TS 23. 228 V8.2.0.

[2] F. Gouveia, T. Magedanz, R. Good, and N. Ventura, "The role of open IMS testbeds in complex service delivery platforms," AFRICON 2007, pp. 1-7, Oct. 2007.

[3] I. Yahia, E. Bertin, J. Deschrevel, and N. Crespi, "Service definition of next generation networks," Proc. of ICNICONMCL 2006, pp. 22-27, Apr. 2006.

[4] M. Melnyk, A. Jukan, and C. Polychronopoulos, "A crosslayer analysis of session setup delay in IP multimedia subsystem (IMS) with EV-DO wireless transmission," IEEE Trans. Multimedia, vol. 9, no. 4, pp. 869-881, June 2007.

[5] Y. Lee, N. Kang, S. Ko, and Y. Kim, "An efficient QoS control mechamism for IMS based convergence network," BCN 2007, pp. 1-12, May 2007.

[6] W. Elleuch, A. Houle, and S. Guenette, "Enabling session mobility in full mesh conferencing model," WiMob 2007 pp. 49-56, Oct. 2007.

[7] G. Camarillo, T. Kauppinen, M. Kuparinen, and I. Ivars, "Towards and innovation oriented IP multimedia subsystem," IEEE Commun. Mag., vol. 45, pp. 130-136, Mar. 2007.

[8] RFC3261 http://www.rfc-editor.org/rfc/rfc3261.txt

[9] P. Podhradsky, E. Mikoczy, O. Labaj, and I. Kotuliak, "NGN platform architecture and its adaptation to the evolution trends," EURASIP Conference 2007, pp. 331-334, May 2007.

[10] G. Camarillo and M. A. Garcia-Martin, The 3G IP multimedia subsystem (IMS), Wiley, 2006.

[11] 3GPP Definition TS 22. 228 V8.6.0.

[12] 3GPP Definition TS 24. 229 V8.8.0

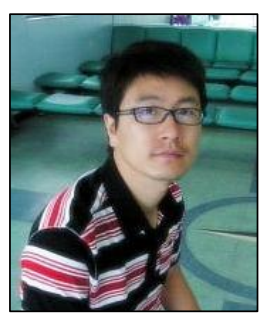

Changho Yun was born in Busan, South Korea on Sept. 23, 1976. He received the M.S. and $\mathrm{Ph}$. D. degrees in department of information and communication from Gwangju Institute of Science and Technology (GIST) in 2004 and 2007, respectively. He was a postdoc researcher in department of Computer Science in North Carolina State univ. Currently, $\mathrm{He}$ is a senior researcher in department of ocean engineering research from Korea Ocean Research \& Development Institute (KORDI). His current research interests include broadband convergence network $(\mathrm{BcN})$, next generation network (NGN), IP multimedia subsystem (IMS), as well as underwater acoustic network (UANet).

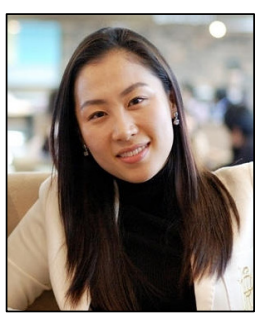

A-Ra Cho was born in Jinhae, South Korea on Oct. 17, 1979. She received the B.S. degree in electronic engineering from the Kyunghee University in 2002. She is a Ph. D. candidate in underwater acoustic communication engineering from the University of Science Technology (UST), and a researcher in the department of ocean engineering research from Korea Ocean Research \& Development Institute (KORDI). Her current research interests include mobile ad-hoc network (MANET) and underwater acoustic network (UANet).

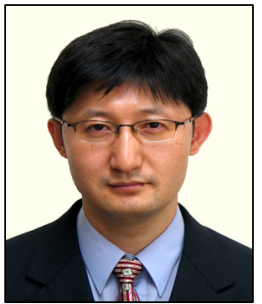

Seung-Geun Kim received the B.S. degree in Electronic Engineering from Inha University, Inchon, Korea, in 1995, and M.S. and Ph.D. degree in Information and Communications from Gwangju Institute of Science and Technology (GIST), Gwangju, Korea, in 1997 and 2002, respectively. Since 2002, he has worked for the Maritime \& Ocean Engineering Research Institute (MOERI), KORDI, as a senior researcher. His research interests include acoustic underwater communication system and maritime communication system design and implementation.

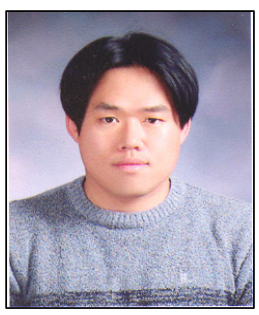

Jong-Won Park was born in Hwasung, South Korea on Feb. 1, 1973. He received the M.S. and $\mathrm{Ph} . \mathrm{D}$. degrees in department of electronic engineering from Ajou University in 1997 and 2006, respectively. Currently, He is a senior researcher in department of ocean engineering research from Korea Ocean Research \& Development Institute (KORDI). His current research interests include underwater robot(AUV, ROV) system, maritime logistics, ship's navigation system, integrated network for ship and naval vessels, as well as underwater acoustic communication and network (UANet).

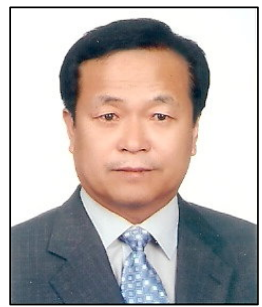

Yong-Kon Lim received the M.S. degrees in power and electrical engineering from the Chung-Nam University in 1984 and $\mathrm{Ph}$. D. degrees in electronic engineering from the AJou University in 1994, respectively. He has been working at Korea Ocean Research \& Development Institute (KORDI) since 1980. Presently, he is the general-director of KORDI and a professor in the department of underwater acoustic communication engineering at University of Science and Technology (UST) and an affiliate professor in that of Ocean Systems Engineering (OSE) at Korea Advanced Institute of Science and Technology (KAIST). His main interests are underwater acoustic telecommunication, underwater acoustic network, integrated logistic system for Ocean, and integrated communication system for ships. 